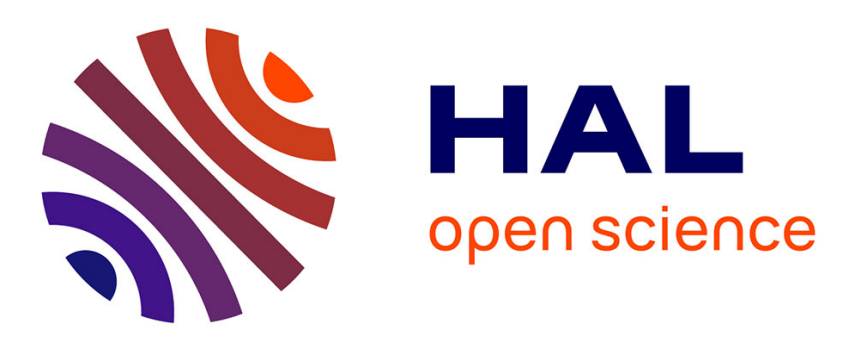

\title{
A framework towards a socially aware Mobile Robot motion in Human-Centered dynamic environment
}

\author{
Amit Kumar Pandey, Rachid Alami
}

\section{To cite this version:}

Amit Kumar Pandey, Rachid Alami. A framework towards a socially aware Mobile Robot motion in Human-Centered dynamic environment. IEEE/RSJ International Conference on Intelligent Robots and Systems (IROS), 2010, Taipei, Taiwan. hal-01977475

\section{HAL Id: hal-01977475 \\ https://hal.laas.fr/hal-01977475}

Submitted on 10 Jan 2019

HAL is a multi-disciplinary open access archive for the deposit and dissemination of scientific research documents, whether they are published or not. The documents may come from teaching and research institutions in France or abroad, or from public or private research centers.
L'archive ouverte pluridisciplinaire HAL, est destinée au dépôt et à la diffusion de documents scientifiques de niveau recherche, publiés ou non, émanant des établissements d'enseignement et de recherche français ou étrangers, des laboratoires publics ou privés. 


\title{
A Framework towards a Socially Aware Mobile Robot Motion in Human-Centered Dynamic Environment
}

\author{
Amit Kumar Pandey and Rachid Alami
}

\begin{abstract}
For a Mobile Robot to navigate in the HumanCentered environment without providing human with an alien like impression by its motion, it should be able to reason about various criteria ranging from clearance, environment structure, unknown objects, social conventions, proximity constraints, presence of a person as well as human groups etc. A human friendly Robot should neither be over-reactive nor be simple wait and move machine. In this paper we provide different mechanism to facilitate robot for analyzing various criteria associated with decision making process of path planning and a framework for their integration which leads to a 'better' path. For the analysis of local clearance and environment structure, a Voronoi diagram based approach is proposed. Then different set of rules are integrated to treat human and other unknown obstacles differently. In our approach for treating human explicitly robot constructs different sets of regions around human and iteratively converges to a set of points (milestones), using social conventions, human proximity guidelines and clearance constraints to generate a smooth path. Once equipped with such capabilities, our robot is able to do higher level reasoning for dynamically deciding about adapting or ignoring a social convention in a particular segment of the environment. This will lead the robot to be aware about its own behavior related to its motion.
\end{abstract}

\section{INTRODUCTION}

A $\mathrm{S}$ Robots have started navigating around us, it is apparent that social norms and reasoning about space around human should be reflected in the robot's motion. In the context of Human-Robot Co-existence with a better harmony, it is necessary that Human should no longer be on the compromising side. Robot should 'equally' be responsible for any compromise, whether it is compromising the shortest path for respecting social norms or compromising the social norms for physical comfort of the person, in the context of navigation in human presence.

In [15], we have evaluated the long term performance of our tour guide robot Rackham with 8000 visitors of the Museum for 16 weeks. Such of our user studies revealed that approaching or avoiding a person as a mobile object with no other consideration is neither enough nor accepted.

In this context it is also important that robot should be able to do a higher level reasoning for planning its path

Authors are with LAAS/CNRS, 7 Avenue de Colonel Roche, 31077, Toulouse, France and University of Toulouse, France.

Email: \{akpandey,rachid.alami\}@laas.fr

The work described here has been partially conducted within the EU Integrated Project CHRIS (Cooperative Human Robot Interaction Systems) [26], funded by the E.C. Division FP7-IST under Contract 215805. based on the local structure of the environment, clearance around human, intended motion of the human and obviously the social conventions of the country it is 'working' in.

In [5], the robot tries to maintain itself in a social queue. In [2], a user study has been conducted for a mobile robot to follow a person in a socially acceptable manner. It concludes that direction following behaviour is more human like and natural than the path following behaviour. In [6], a strategy for passing a person in hallway by a mobile robot has been presented by respecting personal distances. In [16] robot tries to accompany a person by predicting the person's future trajectory. In [17], robot tries to show less reactive and more intuitive behaviour by anticipating the likely action performed by the human based on motion pattern. In [1], a scenario of multiple robots guiding a group of people is presented whereas in [19], the scenario of guiding a visitor to the desired staff member has been addressed but from the viewpoint of reliable person tracking. In [32] the robot tries to behave human like by maintaining 'proper' orientation and distance, while approaching and joining a group of people. In [27], study of adjusting the robot's velocity around the human has been performed. In [39], an approach for co-ordinating motion with the accompanying person has been developed. In [34], we have presented an approach for planning to navigate in the human presence by taking into account costs for obstacles, visibility and hidden areas, which has been further used for placement of the robot in [36] by reasoning on the human perspective. In [35], we have formulated the computation of the velocity profile of robot by taking into account presence and speed of dynamic objects, which might be in the zones hidden from the robot. In [38], we have developed an approach for guiding a person in a social way in the sense it monitors and adapts to the human commitment on the joint task of guiding. In [20], an approach of virtual pedestrian autonomous navigation for crowd simulation has been presented. In it, each agent perceives surrounding agents and extrapolates their trajectory in order to react to potential collisions.

However most of these approaches either assume that the environment structures like corridor, door, hall, etc. are known to the robot or that information is not taken into account at all. Moreover, the set of social conventions the robot should follow are limited and fixed and there is no obvious link with the structure of the local environment. Also the smoothness of the path is not explicitly handled in most of these works.

On the other side, as an attempt to extract topological and semantic meaning from the environment, Voronoi Diagram 
has been shown to be useful by us [40] and by others [30], [8]. Voronoi diagram has been also used for smooth path planning as in [37], [28]. But their approach is to move the robot on the Voronoi diagram itself from the point of view of generating safe and smooth path. However it might add unnecessary overhead in the path length specially while moving in wider free space and moreover it does not explicitly take into account the social conventions and the presence of human in the environment.

Our goal is to develop a mobile robot navigation system which (i) autonomously extract the relevant information about the global structure and the local clearance of the environment from the path planning point of view (ii) dynamically decides upon the selection of the social conventions, which needs to be included at the time of initial planning in different sections of the environment. (iii) at the time of execution, if required, re-plans a smooth deviated path by respecting social conventions (iv) treats an individual, a group of persons and a dynamic or previously unknown obstacle differently.

The framework, which we will present in this paper, basically plans/re-plans a smooth path by interpolating through a set of milestones (the points through which the robot must pass). The key of the framework is the provision of adding, deleting or modifying the milestones based on static and dynamic parts of the environment, the presence and the motion of human or humans' group, the task as well as various social conventions. It also provides the robot with the capability of high level context based reasoning about its motion behaviour.

Keeping oneself on the right side of a narrow passage like corridor, passing by a person from his left side, overtaking a person from his right side, are some widely practiced social rules by human. Moreover, apart from approaching, accompanying, passing by and avoiding differently in different situations, human also tries to maintain an appropriate distance from another human or a group of human even if there is no predicted future collision. And typically human does it while maintaining the smoothness of the path, which is desirable for our robot also for avoiding any feeling of discomfort to the human by its otherwise zigzag motions.

In [31] we have presented an algorithm, which uses a set of social conventions and plans a smooth path to navigate and avoid human. However the information about environment clearance and corridor was already provided to the robot. Also it does not perform the clearance analysis around human, as well as there is no provision to take into account previously unknown obstacles and to re-plan a smooth deviated path for avoiding it.

As far as our knowledge, no significant work has been published, which produces a smooth path by addressing the issues of dynamic \& selective adaptation of social behaviours in a large scale human-centered environment. The rest of this paper will subsequently describe our approach for extracting the path planning oriented environment information. Then the set of social conventions, proximity guidelines and the clearance constraints, within the scope of this paper, will be outlined. Subsequently the concept of selective adaptation of rules will be introduced and their encoding in a decision tree will be discussed. Then the strategies for dealing with dynamic human as well as previously unknown obstacles will be presented, followed by our algorithm to produce the smooth path, with the proof of convergence. Finally the experimental results will be followed by conclusion and pointers for future research and enhancements.

\section{Methodology}

\section{A. Extracting Environment Structure}

As one of our interests is to know the local clearance in the environment like door, narrow passage corridor etc, in our current implementation, we are using Voronoi diagram, which is well known for capturing the skeleton of the environment. Since we are constructing the Voronoi diagram at discrete level of grid cells, we have defined the Voronoi

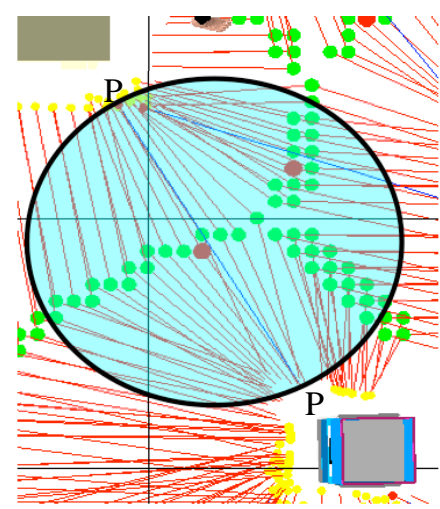

Fig. 1. Extracting Interesting cell (IC) and Interesting Boundary Line, IBL) diagram as the set of cells in the free space that have at least two different equidistant cells in the occupied space. Different Voronoi cells have been shown as small green circles in fig. 1. The lines from each circle connect to the corresponding nearest cells. We define the term 'Interesting

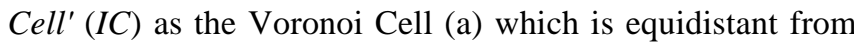
exactly two cells P1 and P2 in the occupied space and (b) both the equidistant points are on the opposite sides on the diameter of the circle centered at the Voronoi point $\mathrm{C}$, such as $\angle P_{1} C P_{2}$ is 180 degrees. We name the line joining both the equidistant points of IC as the 'Interesting Boundary Lines' $(I B L)$. The length of the IBL will be the 'Clearance' of that local region. By setting a threshold on this clearance, robot decides whether it is a narrow passage or wide region. The small red circle ' $\mathrm{C}$ ' is the Voronoi cell, which satisfies the criteria of Interesting cell (IC). Fig. 10 shows the local clearance of the map of our lab, captured by this approach. Note that, as shown in the fig. 10, in case of corridor or long but narrow passage, we will have a set of approximately parallel IBLs.

\section{B. Set of Different Rules}

For our current implementation we use following set of rules:

1) General Social Conventions

In [4], social walking behavior has been classified into 3 broad categories: separation, alignment and cohesion. But 
for this paper we consider following set of widely practiced general social rules in a human-centered environment:

(i) Maintain right-half portion in a narrow passage like hallway, door or pedestrian path. (ii) Pass by a person from his left side. (iii) Overtake a person, from his left side. (iv) Avoid very close sudden appearance from behind a wall.

\section{2) General Proximity Guidelines}

Given that proxemics plays an important role in HumanHuman interaction, proxemics literatures typically divide the space around a person into 4 zones [21]: (i) Intimate (ii) Personal (iii) Social (iv) Public distances. Several user studies and experiments, [6], [9] have been performed to establish and/or verify these spatial distance zones from the viewpoint of Human-Robot interaction. Based on these, in [31] we have hypothesized a set of parameterized semielliptical regions around human as shown in the fig 2 . This will be used in our current implementation for the speed range of $0.5 \mathrm{~m} / \mathrm{s}$ to $1 \mathrm{~m} / \mathrm{s}$ for human and the robot.

Although these regions will serve as a reference for most of the cases, we do not consider the values of its parameters as precise and fixed. As studies suggest, these parameters vary from children to adult, context and the task [22] and even with the personality of the person [23].

The set of proximity rules, which we are presently using, are: (i). Do not enter into intimate space until physical interaction is needed. (ii). Avoid entering into personal space if no interaction with human is required. (iii). Avoid crossing over the person if the robot is already in the side-social regions numbered as 3 and 4 in fig 2, in this case pass by the human from his nearest side. One can notice that in some situations rule (iii) can cause conflict with the rule (ii) of the general social rules presented in section A. But such rules are required to overwrite the general social rules when robot is in close proximity of human.

\section{3) General Clearance Constraints}

The social and proximity rules mentioned above do not handle the situations when any of the regions around human is less spacious than other regions or there is no 'sufficient' space for the robot itself to pass through. The clearance analysis takes care of such situations. The following set of clearance rules are used: (i). Avoid passing through a region around human if it has a clearance less than d1. (ii) Maintain a minimum distance $\mathrm{d} 2$ from the walls and obstacles. (iii) Do not pass through an Interesting Boundary Lines (IBL), if its length is less than $d 3$. Obviously the values of $d 1, d 2$ and $d 3$ depend upon the size of the robot.

We will use the term milestone, as a point through which the path of the robot must pass. In the current implementation, out system performs one the following action for each of the rules mentioned above: (i). Insert a new set of milestones in the list of existing milestones. (ii). Modify the positions of a subset of existing milestones. (iii). Verify whether a particular rule is being satisfied on the existing set of milestones or not.

\section{Selective Adaptation of Rules}

From the path planning point of view we will globally divide these rules into two types: Those which need to be included at the time of initial planning, taking into account the static obstacles only. Rules (i) \& (iv) from set 1 and rules (ii) \& (iii) from set 3 falls into this category. The rule (i) of set 1 is important because, it is commonly experienced fact that if a person is not maintaining himself in the correct side in a narrow passage and another person enters into that passage; a conflicting situation arises, as both try to avoid each other simultaneously. Also there will be conflict if someone wants to overtake the robot in the hallway. Rule (iv) of set 1 will try to avoid collision as well as the feelings like surprise or fear. Rules (ii) and (iii) of set 3 are for obvious reasons to avoid moving very close to obstacle or being stuck in a too narrow passage. Other rules related to Human avoidance and dealing with previously unknown objects will be included at the time of path execution as they will be encountered.

This selective adoption of rules is an attempt to balance the tradeoffs between the path which minimizes the time of flight and the path which avoids conflicting, reactive and confusing situations in a human centered environment.

Hence, the subset of rules, which will be used at the planning stage based on the static environment, are 1(i), 1(iv), 3(ii) and 3(iii). And as explained in the sub-section G, these rules will modify the positions of the milestones.

\section{Construction of Decision Tree}

We have constructed a rule based decision tree based on different possible cases for the relative positions of the human and the next milestone in the current path, as well as the clearance of different regions around the human. In case

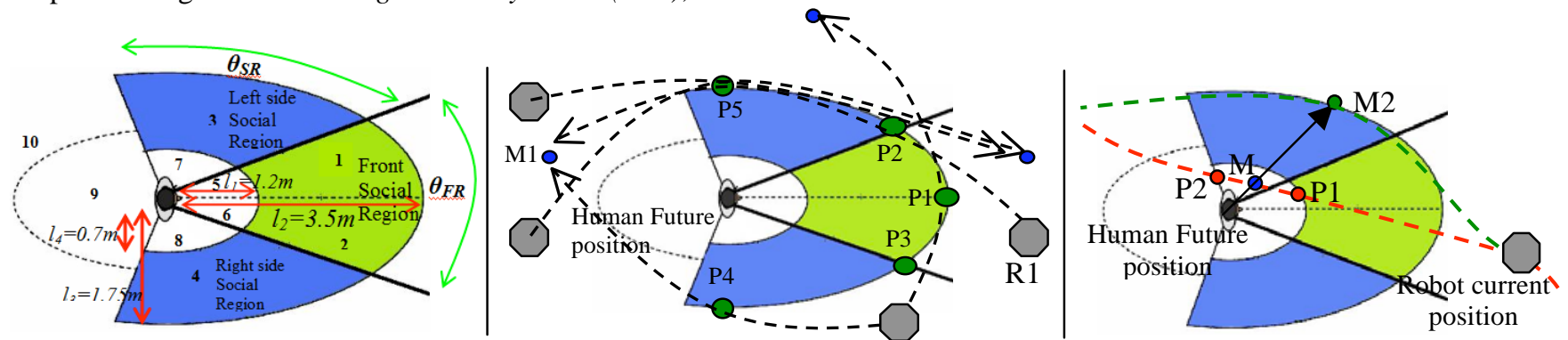

Fig. 2. (a) Construction of parametric region around human. (b) Avoiding a person by using decision tree for getting milestones. Different combination of robot's position (gray polygon) and next milestone of robot's path (blue circle) relative to human predicted position result into different set of points around human (green circles) treated as new milestones for modified path, through which the robot should pass (c) Another way of avoiding a person by calculating new milestone. Initial path (red), modified path (green). The segment P1P2 of the initial path, which intersects the personal space of predicted human future position, is found and its mid point M is projected to point M2 (treated as new milestone) till the social boundary of human. 
of conflicts, the clearance constraints and the proximity guidelines have been given preference over the social conventions in constructing the decision tree. While dealing with the dynamic human, robot uses this decision tree to perform higher-level reasoning. Such decision tree could be learnt by the robot based on the data of human-human avoidance behavior and could be refined by the user studies of robot-human avoidance behavior, which is not the main concern of this paper and is one of our future works.

We define following two functions to query the decision tree:

$($ side, valid_regions $)=g e t \_s i d e \_r e g i o n s\left(R \_p o s, H[i] \_p o s\right.$,

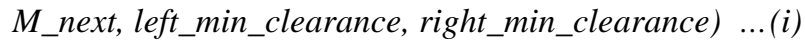

$($ milestones $)=g e t \_m i l e s t o n e s\left(R \_p o s, \quad H[i] \_p o s, \quad M \_n e x t\right.$, side, valid_regions) ...(ii)

where $R \_p o s$ is the current position of the robot, $H[i] \_p o s$ is the predicted position and orientation of the human $i$, $M \_n e x t$ is the immediate next milestone in the robot's current path, left_min_clearance and right_min_clearance are the minimum lengths of interesting boundary lines (IBL) on left and right sides of human predicted position. Function (i) returns, from which side of the human (left, right) the robot should ideally pass and what are the set of acceptable regions (among 1-10, marked in fig 2(a)) around the human, though which the robot could pass. Function (ii) returns subset of points as the intermediate milestones, from the fixed set of points $(P 1, P 2, P 3, P 4, P 5)$ of fig. 2(b), in the order of their passing position, by using the results of function (i).

In fig. 2(b), a subset of the decision tree, in form of different combinations of robot positions (gray) and positions of the next milestone (blue), has been shown. For example, if the robot position is $R 1$, the next milestone to pass through is $M 1$, then the function (i) will return (left, 1, 2,3 ) as the preferred side and acceptable regions in which robot could navigate around human to follow the social norm of passing by. And function (ii) will return $(P 2, P 5)$ as an ordered set of intermediate milestones through which, the path of robot should preferably pass. On the other hand if there would have been some obstacles on the left to the human and left_min_clearance based on IBL around human was not sufficient, the functions would return (right, 2, 4) and (P3, P4).

\section{E. Dealing with Dynamic Human}

As soon as a human becomes visible to the robot and falls within some minimum distance, robot has to decide whether or not to initiate the analysis of human avoidance process. For this robot finds the minimum clearance around the human's predicted future position by constructing a separate set of Interesting Boundary Lines (IBLs), as explained in the section IIA. Robot also predicts a series of future positions for every visible human, by interpolating their previous positions and speeds (studies and works on human walking pattern like [24], [20], could help in better predicting human future trajectory). Then robot checks, whether any segment of its current path is falling inside any of the regions from 1-
9 of fig. 2(a) or not. If not, then the robot will not show any reactive behaviour assuming it will be far from the human and its motion behaviour will not influence human. Otherwise, there will be two cases: some segment of the path falls inside the personal space of the human (5-8) or only inside the social space around human (1-4). In the first case robot will decide to smoothly deviate from its path by replanning, even if there will be no point to point collision with any of the human. This will serve the purpose of maintaining a comfortable social distance from human as well as to signal the human about its awareness and intention well in advance. In the second case robot will first query the decision tree through function (i), get_side_regions(), and checks whether the passing by side returned by the function is same as passing by side while following the current path or not. If not, only then the robot will decide to re-plan the path to comply with the decision tree, to obey various rules.

In any case after taking decision to deviate, robot has to find a set of fixed points (milestones) around human through which the deformed path should pass. For this Fig. 2(c) shows a situation in which the current path of the robot (red line) inters into the personal space of the human predicted position at $P 1$ and exits at $P 2$. Robot first finds the mid point of the line $P 1 P 2$ and projects it to the outer ellipse of social space, from the viewpoint of human predicted future position, at $M 2$. If position of $M 2$ complies with the values returned by function (i), get_side_regions(), it will be used as the milestone to pass through for avoiding the person. Otherwise the robot will use the function (ii), get_milestones(), described in the previous sub-section, to have the milestones for deviation from the fixed set of points around the human, which obey all the constraints.

\section{F. Dealing with Previously Unknown Obstacles}

The obstacles, which were not present in the map provided to the robot or their positions have been changed, need to be dealt dynamically by the robot. For this, robot simply reconstructs Voronoi diagram in a window of width $w$ around the detected obstacle by marking the cells falling inside the bounding box of the obstacle, as occupied. Then for avoiding such obstacles, the rules, which have been discussed in subsection II-C, for planning using static environment, will be used to add or modify milestones for re-planning the smooth deviated path.

\section{G. Generating Smooth Path}

For the current discussion, the task of the robot is to reach to a goal place from its current location. The algorithm to generate the smooth path is as follows:

\section{START; Set FIRST_ITERATION=TRUE}

2 Insert the start and goal points in set of fixed milestones FM.

3 Set $F M \_D=N U L L$; It is set of milestones due to dynamic environment, will be populated at step 17 .

4 Merge the sets of $F M \_D$, in $F M$. Let $t m p \_F M=F M$.

5 Passing through all the milestones of $t m p \_F M$, in the order, plan initial shortest path by cost grid based $\mathrm{A}^{*}$ 
algorithm by taking into account static obstacles only.

the corresponding crossing points $C P$, through which the shortest path $S P$ is passing.

7 If (FIRST_ITERATION $==$ TRUE)

7.1 \{ Extract the information about passing through a narrow passage or corridor.

7.2 If narrow passage or door, mark the corresponding boundary $C B$ as ' $D$ '.

7.3 If corridor, find the entry and exit boundary lines $B C 1$ and $B C 2$ of corridor.

7.4 Remove all the $C B$ s and $C P$ s between $B C 1$ and $B C 2$ from the list of $C B$ and $C P$ and mark them as 'Dead', which will be not used for finding $C B$ in next iteration. Mark $B C 1$ and $B C 2$ as ' $C_{-}$Enter' and ' $C$ E Exit'. Note if robot is already inside a corridor, the $B C l$ will be that $I B L$ of the corridor, which is just next to the robot along the shortest path to the goal.

\subsection{FIRST_ITERATION=FALSE $\}$}

8 Apply the rules set $S R \_P$, which have been selected to be used in planning stage itself, on $C P$. This will shift some of the crossing points marked as 'C_Enter', ' $C \_$Exit' and ' $D$ ' along either side of the corresponding boundary lines.

9 Store the set of modified crossing points in $C P \_M$. Note that $C P{ }_{-} M \subseteq C P$.If $C P \_M=N U L L$, jump to 12 .

10 Set $t m p \_F M=N U L L ;$ Merge $F M$ and $C P \_M$ into tmp_FM.

11 Repeat from step 5.

12 Merge the set $C P$ into the $F M$.

13 Generate Hermite polynomial based smooth spline path by interpolation through $F M$

14 Update the list of visible human, $H$. Find human groups $H G$ and individual human $H I$.

15 For any visible $H G$, if Test for Human Group Avoidance is passed, modify the parameters of elliptical region according to the spread of the group. goto step 17 else goto 18.

16 For any visible HI, if Test for Individual Human Avoidance is passed, goto step 17 else goto step 18.

17 Extract milestones for human group or human avoidance and merge in $F M \_D$.

18 Repeat from step 4.

19 End

The first iteration flag is set to false after the first iteration of the algorithm, to ensure that the robot will pass through the regions and boundaries through which the shortest path is passing, by taking into account the static environment. This will ensure that, just to avoid dynamic objects and human, robot should not take a longer path through entirely different regions Wherever merging in order has been mentioned, it is done by the analysis: between which two successive boundaries of $C P$ a particular point is falling and in the case of conflict the nearer one to the robot is put first in the merged list.
Fig. 3 illustrates different steps of the algorithms. The dotted blue line shows the shortest path from start point $S$ to the goal point $G$, generated by cost grid based A* approach. The Voronoi Diagram of the environment, generated by taking into account the static obstacles only has been shown as skeleton of green points. The thin red lines are the Interesting Boundary Lines (IBL). Reader should not be confused with the rectangular tiles on the floor with IBLs of the map. The blue circles show the set of initial milestones $\mathrm{CP}$, extracted at the first iteration of steps 1-7.5. Now to realize the social rule and clearance constraints selected to be used at the initial planning state as discussed in section II(C), a process of refinement on the milestone along the line of minimum clearance i.e. IBL will be performed. Step 8 performs these refinements on the milestones. For the realization of rule (i), the refinement process is to shift the milestones, which are of a corridor, a door or a narrow opening, towards the middle of the right half portion, i.e. either at boundary_length/4 or at boundary_length*3/4, by calculating which end will be at right side, based on the expected orientation at crossing points. Hence the green milestones at boundaries $1,5,6$ and 7 are obtained by shifting the blue milestones towards the right side of the corridor and door. The refinement associated with other rules are, if the distance of the crossing point is less than a required minimum distances from the nearest end of the corresponding IBL, then shift away the crossing points along the boundary line to maintain this distance. But if boundary is very narrow so that it does not satisfy the minimum threshold, then shift the crossing point at the middle of the boundary. These rules resulted into the green milestones at boundary $3 \quad \& \quad 4$ by shifting the corresponding blue milestones. All the milestones which will be refined by the initial social rules will be treated as the fixed milestones for the next iteration. Steps 9-11 are required to assure the shortest path between two fixed milestones, because few milestones have been shifted so the other milestones may no-longer fall on the probable shorter path as the case with blue milestone of boundaries $2 \& 8$, which has been shifted

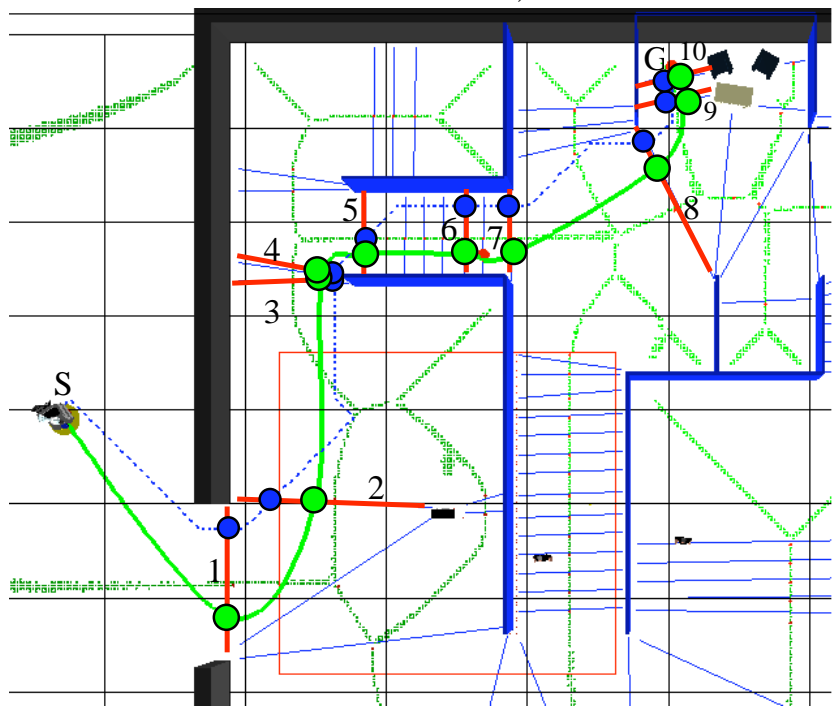

Fig. 3. Steps of iterative refinements on the path to incorporate social conventions and clearance constraints at planning stage. 
to the green milestones in the second iteration of the algorithm.

After having a set of milestones through which the robot should pass, robot uses piecewise cubic spline to connect the points. We are not presenting the equations here, but we use Hermite cubic polynomial and solve it for boundary conditions with the continuity constraint on velocity and acceleration. The green curve in fig. 3 shows the final smooth path generated by using final set of milestones.

\section{H. Proof of Convergence}

The guarantee of the convergence of the algorithm lies in the fact that, after each iteration, it will have a set of fixed milestones, which will not change in next iterations, as they will already be satisfying the rules. Hence eventually the step 9 will result into an empty set of modified milestones, $C P \_M$, and will jump to step 12 to generate the final path. In all our test runs, in 2-3 iterations the algorithm has converged hence facilitating the algorithm to run online.

\section{EXPERIMENTAL RESULTS AND ANALYSIS}

We have tested our framework both in simulation and on real robot Jido. For the tests in simulation, we feed the models of environment, robot and human in our developed robotics software platform Move3D [25]. The inset inside the red box in Fig. 4 is a big simulated environment of dimension $25 \mathrm{~m} \times 25 \mathrm{~m}$. Fig. 4 shows the portion containing the start and goal positions of the robot. The blue lines are the Interesting Boundary Lines (IBLs) extracted by our proposed approach. The voronoi diagram has been drawn as dotted curves. The shortest path has been shown as blue dotted lines. The green curve is the smooth path generated by the robot by our proposed algorithm. Note that robot was able to correctly infer that it is in a corridor and it has shifted the path to the right side of the corridor, till the autonomously found exit of the corridor. On close

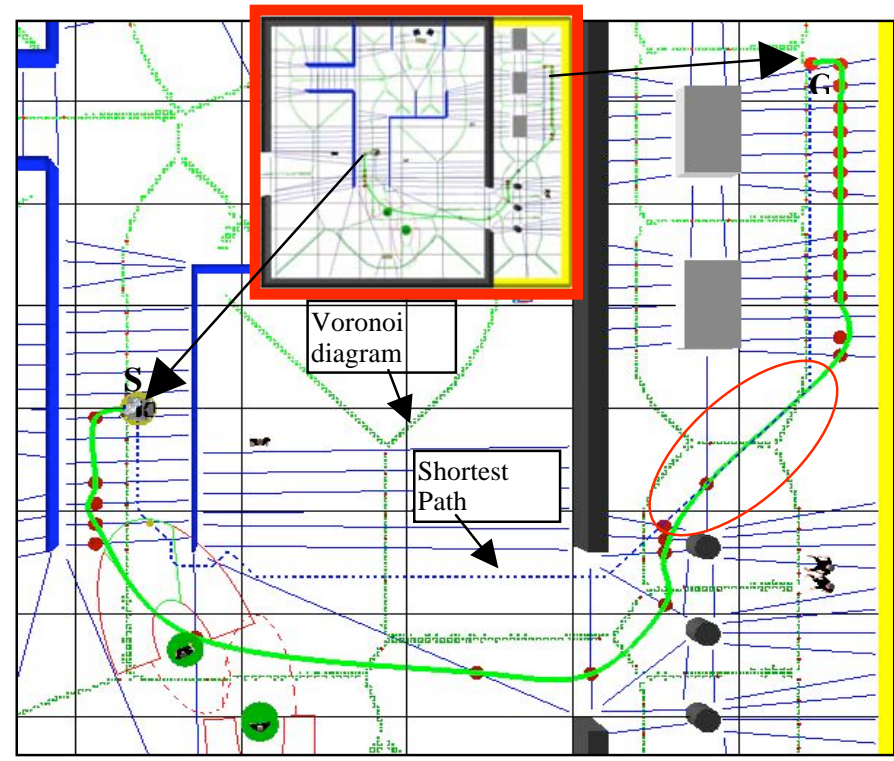

Fig. 4. (Thick Green curve) Smooth and socially acceptable path planned by our approach. (Dotted Blue Line) shortest path planned by cost grid based approach. (Green skeleton of points) Voronoi diagram. comparison of our planned path with Voronoi diagram as well as with the shortest path, one can find that our approach avoids unnecessary route of Voronoi diagram in a wider regions and maintaining the social conventions and other constraints. Whereas, in the regions where all the constraints are satisfied, our algorithm automatically sticks to the shortest path, for example the region enclosed by the red ellipse. But also whenever there is no sufficient clearance in a door or corridor, our algorithm will shift the crossing points to the middle of the boundary, hence following the Voronoi diagram in that region. Hence our algorithm inherits the characteristics of both at the places where they perform better as well as globally maintain the social conventions and smoothness of the path.

Fig. 5(a) shows robot passing by a person in the corridor without showing any unnecessary reactive behavior. Fig. 5(b) and 5(c) show the detection and avoidance a group of people from the left side of the group. Note the smooth deviation in the path in fig. 5(b) at the predicted passing by place, compared to the path in fig. 4. Based on the speed and position of humans, robot autonomously detects a group of people moving together and constructs a unified region by modifying the parameters fig. 2(a) based on the spread of the group (details of which are not provided for the conciseness) Then using our proposed framework, it finds the milestones to modify the path in a socially acceptable manner.

We have implemented our presented framework on our mobile robot Jido. It is equipped with front and back laser and two pairs of cameras. For detecting dynamic objects like trash bin, table, chair it uses vision based module which detects and identifies the objects by its visual tag. However for reliable detection of people, we use the motion capture system installed in the environment, which provides the $3 \mathrm{D}$ information of markers attached with the cap on the human head, which robot uses to compute human position online. Fig. 6 shows the sequence of images where the robot has detected the person and modified its path to smoothly avoid

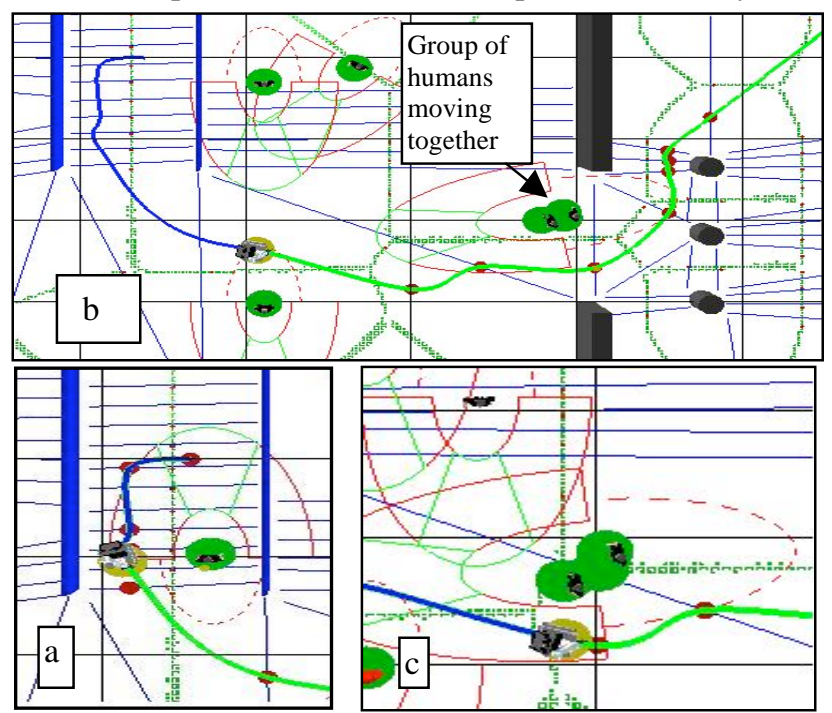

Fig. 5. Continued from fig. 4. (a) Robot passing by a person in corridor, without any reactive behavior, by maintaining social norm. (b) Robot planned a smooth deviation in path to avoid a group of people. (c) Smoothly avoiding the group by passing by from left. 


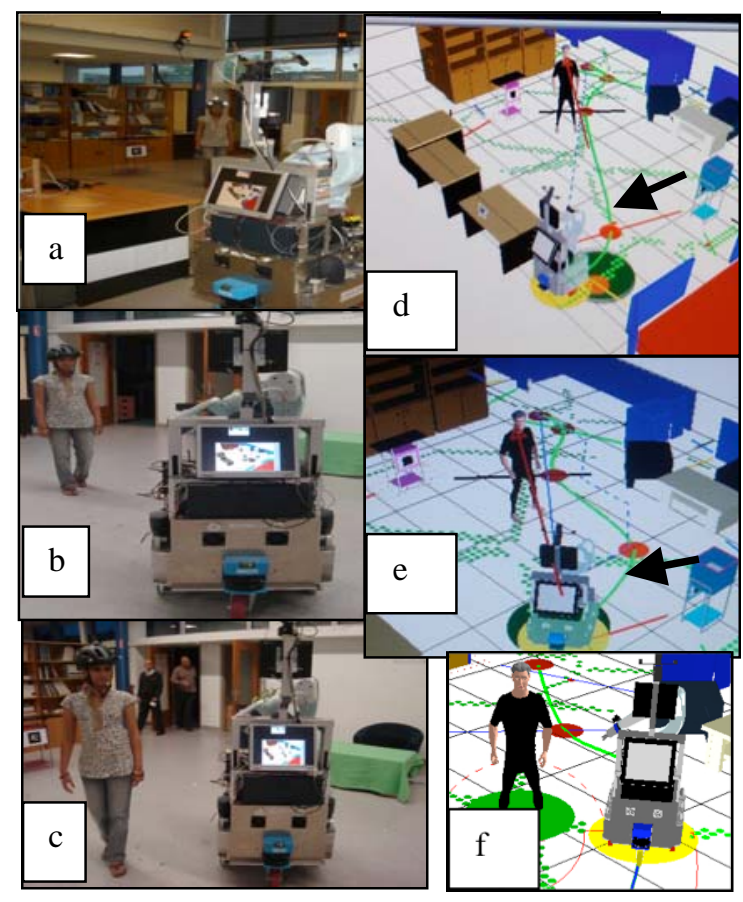

Fig. 6. Our experimental mobile robot Jido, avoiding the person by maintaining the social convention of passing by from her left side.
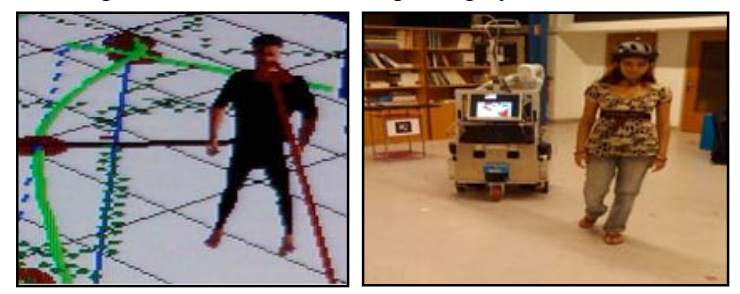

Fig. 7. Suspending the social convention of passing by from left and passing from right side because of insufficient clearance on the left side of human.
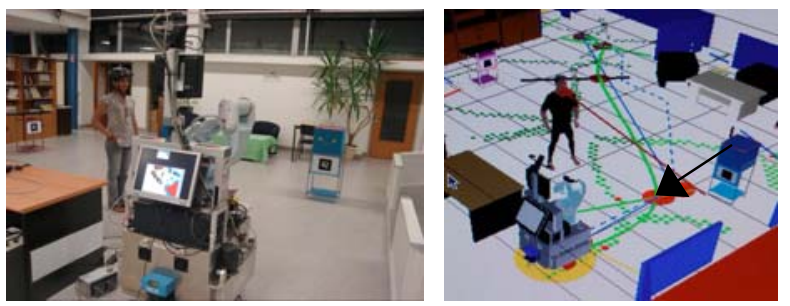

Fig. 8. Crossing a standing person, avoids entering into personal space. the person from her left side. Fig. 6(d) (e) show the current state of the environment which robot is maintaining and the black arrows show the initial and the modified path of the robot after it has predicted that even if there is no direct collision it might enter into personal space of the human. Fig. 7 shows the case where robot has suspended the social convention of passing by, as the clearance was not sufficient on the left side of the person. Instead the robot has modified its path to pass by from the right side of the human for avoiding entering into the personal space. Fig. 8 shows the case when the human is not moving. Robot has planned the path, shown as black arrow, to smoothly cross the person to reach the goal, while maintaining the proximity constraints around the person.

Fig. 9 shows the results of avoiding previously unknown obstacles, for which robot updates the Voronoi diagram to extract the clearance information and our presented
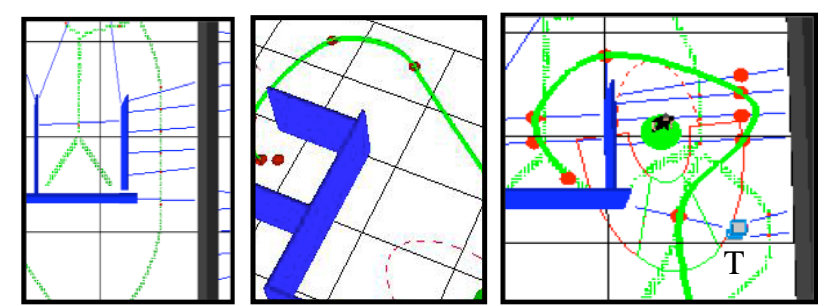

Fig. 9. (a) Initial Voronoi Diagram and clearance (IBLs), (b) Initial planned path, (c) During the execution time, updating clearance information and replanning due to presence of previously unknown trash bin, marked as $\mathrm{T}$.

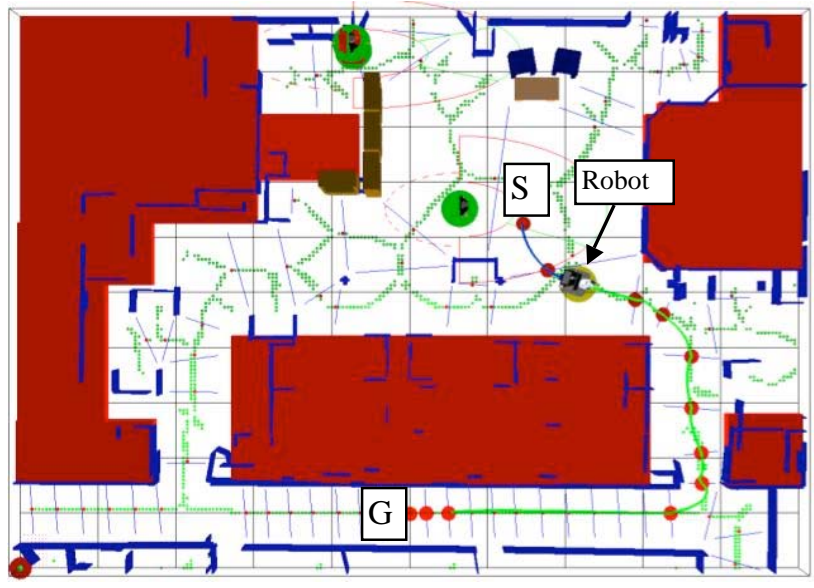

Fig. 10. Path generated in the bigger map of our lab, from $\mathrm{S}$ to $\mathrm{G}$

algorithm adds new sets of milestones as shown in fig. 9(c), and re-plan the smooth deviated path.

Fig. 10 shows the bigger portion of our lab having corridor. The green curve is the smooth path generated by the presented approach for the robot to reach from $\mathrm{S}$ to $\mathrm{G}$.

Although our implementation is generic enough to easily switch to the left handed walking rule for avoiding persons and moving in corridor, we will not show these results here.

\section{CONCLUSION AND FUTURE WORKS}

This paper is a step towards enabling a robot socially and contextually aware. The key idea of the presented framework is to get a set of milestones and plan a smooth path through them. Our framework facilitates the addition, deletion or modification in the milestone based on various rules. The generality of the framework lies in the fact that, any task or rule, which could result into some milestones or could perform some operation on existing milestones could be easily incorporated. For example in [38] we have presented milestone-based approach for guiding a person in a socially acceptable manner; hence the task of guiding could also be easily incorporated in the presented framework. We are in the process of also incorporating the tasks of approaching and accompanying a person. We have also presented method to practically extract the clearance information in the grid-based map of the environment. Then we have presented the concept of selective adoption of rules. Our robot equipped with such capabilities autonomously decides, which conventions needs to be used in a particular part of the environment and at a particular state of planning and execution. We have also shown that apart from 
satisfying various constraints, the generated path inherits the characteristics of both, the cost grid based shortest path as well as Voronoi diagram based path at the places, where they perform better. Moreover our approach treats an individual, a group of humans and an unknown obstacle differently. We have also presented the proof of convergence of our algorithm.

This robotics platform could also be used for various user studies. However our present work is germinated from our past user studies [15], [33], more user studies is absolutely necessary including emotional and psychological responses. It will also refine and evolve the parameters of the decision tree. Such rigorous user studies will not only be useful to verify the correlation among various factors like social convention, proximity and clearance but also to identify various other factors and missing links, which define behavior and shape of the path in navigation [20].

\section{REFERENCES}

[1] E. Martinez-Garcia, O. Akihisa, and S. Yuta, "Crowding and guiding groups of humans by teams of mobile robots," in IEEE Workshop on Advanced Robotics and its Social Impacts, ARSO, Nagoya, Japan, 2005, pp. 91-96.

[2] R. Gockley, J. Forlizzi, and R. Simmons, "Natural person-following behavior for social robots," in Proceedings of the ACM/IEEE HRI, Washington DC, USA, 2007, pp. 17-24.

[3] V. Kulyukin, C. Gharpure, J. Nicholson, and G. Osborne, "Robot assisted wayfinding for the visually impaired in structured indoor environments," Autonomous Robots, vol. 21, no. 1, 2006.

[4] B. Martens and A. Brown, "Simulating Human Behaviour in Built Environments," In Springer Computer Aided Architectural Design Futures, pp. 301-310, 2007.

[5] Yasushi Nakauchi and Reid Simmons, "A Social Robot that Stands in Line," Autonomous Robots, vol. 12, pp. 313-324, 2002.

[6] E. Pacchierotti, H. Christensen, and P. Jensfelt, "Human-robot embodied interaction in hallway settings: a pilot user study," in IEEE RO-MAN, Nashville, USA, 2005, pp. 164-171.

[7] S. Thrun, J. Schulte, and C. Rosenberg, "Interaction with mobile robots in public places," IEEE Intelligent Systems, pp. 7-11, 2000.

[8] Sebastian Thrun, "Learning metric-topological maps for indoor mobile robot navigation," Artificial Intelligence, vol 99, no 1, pp. 2171, February 1998.

[9] M. Yoda and Y. Shiota, "The mobile robot which passes a man," IEEE International workshop on robotics and Human Robot Communication, pp. 112-117, 1997.

[10] S. Nishimura, H. Takemura, and H. Mizoguchi, "Development of attachable modules for robotizing daily items -person following shoppingcart robot-," IEEE Robotics and Biomimetics, 2007.

[11] H. Asoh, Y. Motomura, F. Asano, I. Hara, S. Hayamizu, K. Itou,T. Kurita, T. Matsui, N. Vlassis, R. Bunschoten, and B. Krose, "Jijo-2: an office robot that communicates and learns," IEEE Intelligent Systems, vol. 16, no. 5, 2001.

[12] A. Clodic, M. Ransan, R. Alami, and V. Montreuil, "A management of mutual belief for human-robot interaction," in IEEE International Conference on Systems, Man and Cybernetics, ISIC, Montreal, Canada, 2007, pp. 1551-1556.

[13] P. R. Cohen and H. J. Levesque, "Intention is choice with commitment," Artificial Intelligence, vol. 42(2-3), pp. 213-261, 1990.

[14] E. Prassler, D. Bank, and B. Kluge, "Motion coordination between a human and a mobile robot," in IEEE/RSJ IROS, 2002, pp. 1228-1233.

[15] A. Clodic, S. Fleury, R. Alami, R. Chatila, G. Bailly, L. Brethes, M. Cottret, P. Danes, X. Dollat, F. Elisei, I. Ferrane, M. Herrb, G. Infantes, C. Lemaire, F. Lerasle, J. Manhes, P. Marcoul, P. Menezes, and V. Montreuil, "Rackham: An interactive robot-guide," RO-MAN, pp. 502-509, 2006.

[16] F. Hoeller, D. Schulz, M. Moors, and F. Schneider, "Accompanying persons with a mobile robot using motion prediction and probabilistic roadmaps," in IEEE/RSJ IROS, San Diego, 2007, pp. 1260-1265.
[17] M. Bennewitz, W. Burgard, and S. Thrun, "Adapting navigation strategies using motions patterns of people," in IEEE ICRA, vol. 2, Taipei, Taiwan, 2003, pp. 2000-2005.

[18] R. Gockley, J. Forlizzi, and R. Simmons, "Natural person-following behavior for social robots," in Proceedings of the ACM/IEEE HRI, Washington DC, USA, 2007, pp. 17-24.

[19] C. Martin, H. Bohme, and H. Gross, "Conception and realization of a multi-sensory interactive mobile office guide," IEEE Systems, Man and Cybernetics, vol. 6, 2004

[20] S. Paris, J. Pettré, and S. Donikian, "Pedestrian Reactive Navigation for Crowd Simulation: a Predictive Approach" Eurographics: Computer Graphics Forum, Volume 26, Number 3, pp 665-674, 2007

[21] E. T. Hall, The Hidden Dimension. N.Y.: Doubleday, 1966.

[22] F. Yamaoka, T. Kanda, H. Ishiguro, and N. Hagita, "How close?: model of proximity control for information-presenting robots," in ACM/IEEE international conference on HRI, Amsterdam, The Netherlands, 2008, pp. 137-144.

[23] M. Walters, K. Dautenhahn, R. te Boekhorst, K. L. Koay, C. Kaouri, S. Woods, C. Nehaniv, D. Lee, and I. Werry, "The influence of subjects' personality traits on personal spatial zones in a human-robot interaction experiment," in IEEE RO-MAN, Nashville, USA, 2005.

[24] G. Arechavaleta, J.-P. Laumond, H. Hicheur, and A. Berthoz, "On the nonholonomic nature of human locomotion," Autonomous Robots, vol. 25, pp. 25-35, 2008.

[25] T. Simeon, J.-P. Laumond, and F. Lamiraux, "Move3d: A generic platform for path planning," in IEEE International Symposium on Assembly and Task Planning, Fukuoka, Japan, 2001, pp. 25-30.

[26] “http://www-iri.upc.es/groups/urus/," European FP6 URUS Project.

[27] D. Shi, E. G. Collins Jr., A. Donate, X. Liu, B. Goldiez, and D. Dunlap, "Human-aware robot motion planning with velocity constraints," in International Symposium on Collaborative Technologies and Systems, May 2008, pp 490-497.

[28] S. Garrido, L. Moreno, and D. Blanco, "Voronoi diagram and fast marching applied to path planning," in IEEE ICRA, Orlando, Florida, 2006, pp. 3049-3054.

[29] S. Garrido, L. Moreno, and D. Blanco, "Exploration of a clustered environment using voronoi transformation and fast marching," Robotics and Autonomous Systems, vol 56, pp. 1069-1081, 2008.

[30] S. Friedman, H. Pasula and D. Fox, "Voronoi random fields: extracting the topological structure of the indoor environment via place labeling," IJCAI, Hyderabad, India, 2007, pp. 2109-2114.

[31] A. K. Pandey and R. Alami, "A framework for adapting social conventions in a mobile robot motion in human-centered environment," in $14^{\text {th }}$ International Conference on Advanced Robotics, Munich, Germany, 2009.

[32] P. Althaus, H. Ishiguro, T. Kanda, T. Miyashita, and H. I. Christensen, "Navigation for human-robot interaction tasks," in IEEE ICRA, New Orleans, LA, 2004, pp. 1894-1900.

[33] K.L. Koay, E. A. Sisbot, D. A. Syrdal, M.L. Walters, K. Dautenhahn and R. Alami, "Exploratory Study of a Robot Approaching a Person in the Context of Handling Over an Object," AAAI Spring Symposia (AAAI 07), Palo Alto, California, USA, 2007.

[34] E. A. Sisbot, L. F. Marin-Urias, R. Alami and T. Simeon, "A Human Aware Mobile Robot Motion Planner," IEEE Transactions on Robotics, Vol 23, Issue: 5, pp. 874-883, 2007.

[35] K. M. Krishna, R. Alami and T. Simeon, "Safe proactive plans and their execution", Robotics and Autonomous Systems, Vol 54, Issue 3, pp. 244-255, 2006.

[36] L. F. M-Urias, E. A. Sisbot and R. Alami, "Geometric tools for Perspective Taking for Human-Robot Interaction," 7th MICAI,exican International Conference on Artificial Intelligence, Mexico 2008.

[37] A. C. Victorino, P. Rives, J-J. Borrelly, "Safe Navigation for Indoor Mobile Robots. Part I: A Sensor-Based Navigation Framework," International Journal of Robotics Research, Vol. 22, No. 12, pp.1005$1118,2003$.

[38] A. K. Pandey and R. Alami, "A step towards a sociable robot guide which monitors and adapts to the person's activities," $14^{\text {th }}$ International Conference on Advanced Robotics, Germany, 2009.

[39] E. Prassler, D. Bank, and B. Kluge, "Motion coordination between a human and a mobile robot," in IEEE/RSJ IROS, 2002, pp. 1228-1233.

[40] D. V. Zwynsvoorde, T. Simen, R. Alami, "Building topological models for navigation in large scale environments," IEEE ICRA, Seoul, 2001, pp.4256-4261. 